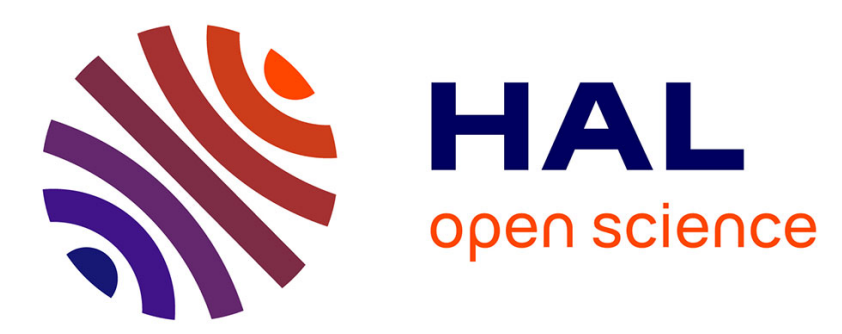

\title{
On the structure of polyhedral positive invariant sets with respect to delay difference equations
}

Mohammed-Tahar Laraba, Sorin Olaru, Silviu-Iulian Niculescu

\section{To cite this version:}

Mohammed-Tahar Laraba, Sorin Olaru, Silviu-Iulian Niculescu. On the structure of polyhedral positive invariant sets with respect to delay difference equations. Advances in Difference Equations and Discrete Dynamical Systems. ICDEA 2016, 2017. hal-01817035

\section{HAL Id: hal-01817035 \\ https://hal-centralesupelec.archives-ouvertes.fr/hal-01817035}

Submitted on 16 Jun 2018

HAL is a multi-disciplinary open access archive for the deposit and dissemination of scientific research documents, whether they are published or not. The documents may come from teaching and research institutions in France or abroad, or from public or private research centers.
L'archive ouverte pluridisciplinaire HAL, est destinée au dépôt et à la diffusion de documents scientifiques de niveau recherche, publiés ou non, émanant des établissements d'enseignement et de recherche français ou étrangers, des laboratoires publics ou privés. 


\title{
On the structure of polyhedral positive invariant sets with respect to delay difference equations
}

\author{
Mohammed-Tahar Laraba, Sorin Olaru and Silviu-Iulian Niculescu
}

\begin{abstract}
This chapter is dedicated to the study of the positive invariance of polyhedral sets with respect to dynamical systems described by discrete-time delay difference equations DDE. Set invariance in the original state space, also referred to as $\mathscr{D}$-invariance, leads to conservative definitions due to its delay independent property. This limitation makes the $\mathscr{D}$-invariant sets only applicable to a limited class of systems. However, there exists a degree of freedom in the state-space transformations which can enable the positive invariant set-characterizations. In this work we revisit the set factorizations and extend their use in order to establish flexible set-theoretic analysis tools. With linear algebra structural results, it is shown that similarity transformations are a key element in the characterization of low complexity invariant sets within the class of convex polyhedral candidates. In short, it is shown that we can construct, in a low dimensional state-space, an invariant set for a dynamical system governed by a delay difference equation. The basic idea which enables the construction is a simple change of coordinates for the DDE. The obtained $\mathscr{D}$-invariant set exists in the new coordinates even if its existence necessary conditions are not fulfilled in the original state space. This proves that the $\mathscr{D}$-invariance notion is dependent on the state-space representation of the dynamics. It is worth to recall as a term of comparison that the positive invariance for delay-free dynamics is independent of the state-space realization.
\end{abstract}

\section{Introduction}

The construction of positive invariant sets is an essential concept in relationship with stability analysis and in general in control theory with applications in constrained control, uncertainty handling and design problems. It serves as a basic tool in model predictive control [1], fault tolerant control [2] and reference governor design [3].

All the authors are with the Laboratory of Signals and Systems of CentraleSupelec, 91190 Gif-SurYvette, France e-mail: mohammed.laraba; sorin.olaru; silviu.niculescu@12s.centralesupelec.fr 
Two popular constructions of positive invariant sets for delay difference equations exist. The first approach, referred to as Krasovskii approach, makes use of the fact that in discrete-time a finite dimensional extended state space model can be constructed. The study is then simplified in the case of linear discrete time systems as long as the difficulties related to the infinite dimensionality of the state space are avoided. A higher dimensional linear time-invariant system is obtained, its dimension is finite but is in direct relation with the delay value. The equivalent linear time invariant model [4-6] provides an invariant set for the delay difference equation [7]. However, this approach suffers from an increased computational complexity with the delay's size and become impracticable when delays are relatively large. Hence, an alternative approach for the construction of invariant sets for DDEs referred to as Razumikhin approach and denoted as $\mathscr{D}$-invariance has been considered [8]. This approach has been formulated to obtain an invariant set for the DDE in the original state space, which is independent from the delay value. Iterative procedures for the construction of $\mathscr{D}$-invariant sets as well as the relationship between time-varying DDE stability and $\mathscr{D}$-invariance were presented in [9-11]. However, the concept of $\mathscr{D}$-invariance is often conservative as long as the existence conditions are restrictive.

Recently, it has been recognized that $\mathscr{D}$-invariance can be seen from the geometrical point of view as a factorization of invariant set in the extended state space [12]. It has been established that the extended state space invariance corresponds to a minimal factorization while $\mathscr{D}$-invariance, under the constraints imposed by the dimension of the DDE, represents the maximal regular ordered factorization. This interesting result opens the way for factorizations which are in between the two representations by exploiting non-minimal state space equations.

In this chapter, the link between the Razumikhin approach and the Krasovskii approach will be revisited using set factorization. The proposed framework yields a fitting trade-off between the conceptual generality of the extended state space approach and the computational convenience of the $\mathscr{D}$-invariance approach. We show that $\mathscr{D}$-invariance, which can be seen as set factorization of an invariant set in the extended state space, represents a particular realization of a broader family of invariant structures. The relationship between these families of invariant sets is established via set factorization and conjugacy.

After establishing the general result, a numerical example will be detailed for illustration. Therein, a dynamical system with a maximum delay equal to 2 and a state space representation of dimension 2 will be studied. For this delay difference equation, the necessary condition for the existence of $\mathscr{D}$-invariant sets proposed in [13] are not fulfilled. However, we propose a simple similarity transformation, which leads to a regular ordered factorization of the extended-state invariant set, and thus, allows the construction of a $\mathscr{D}$-invariant set in the novel basis. The chapter is organized as follows. Section 2 presents some preliminary definitions and the existing results of [12] are recalled. In section 3, the problem of existence and uniqueness of similarity transformations for the construction of $\mathscr{D}$-invariant sets is addressed. The shape of the similarity transformation which allows a regular ordered factorization is established in section 4. In section 5, a numerical example is given to illustrate the previous results and finally section 6 draws concluding remarks. 


\section{Preliminary definitions and existing results}

Let us consider the following delay difference equation:

$$
x(k)=\sum_{i=1}^{d} A_{i} x(k-i)
$$

where $x(k) \in \mathbb{R}^{n}$ is the state vector at the time $k \in \mathbb{Z}_{+}$. Matrices $A_{i} \in \mathbb{R}^{n \times n}$ for $i=$ $1, \cdots, d$. For every interval $\Pi$ of $\mathbb{R}_{+}$we define $\mathbb{R}_{\Pi}:=\mathbb{R} \cap \Pi$. The initial conditions are considered to be given by $x(-i) \in \mathbb{R}^{n}$, for $i \in \mathbb{Z}_{[1, d]}$. Given two sets $\mathscr{X}, \mathscr{Y} \subset \mathbb{R}^{m}$, $\mathscr{X} \oplus \mathscr{Y}$ and $\mathscr{X} \times \mathscr{Y}$ denote the Minkowski sum and the Cartesian product of these two sets, respectively, defined as follows:

$$
\begin{gathered}
\mathscr{X} \oplus \mathscr{Y}:=\{z \mid \exists(x, y) \in(\mathscr{X}, \mathscr{Y}) \text { such that } z=x+y\} . \\
\mathscr{X} \times \mathscr{Y}:=\{(x, y) \mid x \in \mathscr{X} \text { and } y \in \mathscr{Y}\} .
\end{gathered}
$$

An extended state space representation can be constructed for any given (finite) delay realization. Using an augmented state vector

$$
z(k)=\left[x(k)^{T} \cdots x(k-d+1)^{T}\right]^{T}
$$

equation (1) can be rewritten as:

$$
z(k)=A_{e} z(k-1)=\left[\begin{array}{cccc}
A_{1} & \ldots & A_{d-1} & A_{d} \\
I & \ldots & 0 & 0 \\
\vdots & \ddots & \vdots & \vdots \\
0 & \ldots & I & 0
\end{array}\right] z(k-1)
$$

Definition 1. A set $\mathscr{P} \subseteq \mathbb{R}^{n}$ is called $\mathscr{D}$-invariant for the system (1) with initial conditions $x(-i) \in \mathscr{P}$ for all $i \in \mathbb{Z}_{[1, d]}$ if the state trajectory satisfies $x_{k} \in \mathscr{P}, \forall k \in$ $\mathbb{Z}_{+}$.

Two main approaches exist in the literature dealing with positive invariant sets for discrete-time delay difference equations; the invariant set in an extended state space (2) on one side and the invariant set in the original state space (1) (also called $\mathscr{D}$ invariant set) on the other side. The concept of cyclic invariance [14] proposes instead of a rigid set in $\mathbb{R}^{n d}$ or $\mathbb{R}^{n}$ a family of invariant sets and offers a certain degree of flexibility.

Definition 2. A family of ( $d$ tuples of) sets $\left\{\Omega_{1}, \ldots, \Omega_{d}\right\}$ is called cyclic $\mathscr{D}$-invariant with respect to (1) if:

$$
\begin{gathered}
A_{1} \Omega_{1} \oplus A_{2} \Omega_{2} \oplus \cdots \oplus A_{d} \Omega_{d} \subseteq \Omega_{d} ; \\
A_{1} \Omega_{d_{m}} \oplus A_{2} \Omega_{1} \oplus \cdots \oplus A_{d} \Omega_{d-1} \subseteq \Omega_{d-1} ; \\
\vdots \\
A_{1} \Omega_{2} \oplus A_{2} \Omega_{3} \oplus \cdots \oplus A_{d} \Omega_{1} \subseteq \Omega_{1}
\end{gathered}
$$


A generalization of the cyclic invariance notion to invariant family of sets was proposed by [15].

Definition 3. A family of ( $d$ tuples of) sets $\mathscr{F} \subset\left(\mathbb{R}^{n d}\right.$ is an invariant family with respect to (1) if for any tuple $\left\{\Omega_{1}, \Omega_{2}, \ldots, \Omega_{d}\right\} \in \mathscr{F}$ there exists a set $\Omega_{0} \subset \mathbb{R}^{n}$ such that $\left\{\Omega_{0}, \Omega_{1} \ldots, \Omega_{d-1}\right\} \in \mathscr{F}$ and

$$
A_{1} \Omega_{1} \oplus A_{2} \Omega_{2} \oplus \cdots \oplus A_{d} \Omega_{d} \subseteq \Omega_{0}
$$

The link between the two main representations for discrete-time delay difference equations and their invariant sets has received recently a unifying characterization via set factorization [12]. The reader interested in a more thorough introduction to set factorization may consult [16], [17]. Next, the basic notions in this respect.

A partition of a set of indices $P \subset \mathbb{Z}_{[1, m]}$ is the family of ' $l$ ' subsets $P_{k}$ of $P$, which verify the following conditions :

- $\emptyset \notin\left\{P_{k}\right\}_{k=1}^{l}$

- The subsets $\left\{P_{k}\right\}_{k=1}^{l}$ are said to cover $P$ i.e. $P=\bigcup_{i=1}^{l} P_{i}$

- The elements of $\left\{P_{k}\right\}_{k=1}^{l}$ are pairwise disjoint $\left(P_{i} \cap P_{j}=\emptyset\right.$ for $\left.i \neq j\right)$.

Given a subset $P_{i} \subset \mathbb{Z}_{[1, m]}$ and a set $\Omega \in \mathbb{R}^{m}, \Omega_{\downarrow P_{i}}$ denotes the projection of the set $\Omega$ on the subset of $\mathbb{R}^{n}$ with indices of Cartesian coordinates in $P_{i}$. Before introducing the main concepts it is useful to recall the definition of the Cartesian product of two sets $\Omega_{1}$ and $\Omega_{2}$ as it will be used subsequently.

$$
\Omega_{1} \times \Omega_{2}=\left\{(x, y) \mid x \in \Omega_{1} \text { and } y \in \Omega_{2}\right\} .
$$

Definition 4. Let $\Omega \in \mathbb{R}^{m}$ and $\bigcup_{i=1}^{l} P_{i}$ be the partition of $\mathbb{Z}_{[1, m]}$

1. The set $\Omega$ is factorized according to the partition $\bigcup_{i=1}^{l} P_{i}=\mathbb{Z}_{[1, m]}$ if:

$$
\Omega=\Omega_{\downarrow P_{1}} \times \cdots \times \Omega_{\downarrow P_{l}}
$$

2. A set factorization (5) is said to be balanced if:

$$
\operatorname{card}\left\{P_{1}\right\}=\cdots=\operatorname{card}\left\{P_{l}\right\}
$$

3. A factorization is said to be ordered if it is defined by an ordered partition $P=$ $\bigcup_{k=1}^{l} P_{k}$ satisfying

$$
\max \left\{P_{i}\right\}<\min \left\{P_{j}\right\}, \forall i<j \in \mathbb{Z}_{[1, m]} ;
$$

4. A factorization is regular if is characterized by the equivalence of the factors

$$
\Omega_{\downarrow P_{1}}=\cdots=\Omega_{\downarrow P_{l}}=S
$$


and

$$
\Omega=\underbrace{S \times S \times \cdots \times S}_{l \text { times }}
$$

Most of the factorization properties are related to the Cartesian product operation. It is clear that the set factorization is a non-commutative. The exception is represented by the regular factorization which is commutative inside the given partition. Additionally, the regular factorizations are balanced but not necessarily ordered. From the structural point of view, the geometry of the factors is related to the geometry of the initial set. Convexity, for example, of a given set $\Omega$ implies the convexity of the factors. It is worth to be mentioned that the Cartesian product of several polyhedra is a polyhedron of higher dimension. It becomes clear that the polyhedral sets represent an interesting class of sets which can be used for the development in relationship with set factorization. In comparison, even if the projection of ellipsoidal sets is ellipsoidal, the Cartesian product of ellipsoids is not an ellipsoid rendering the ellipsoidal class of sets impracticable for set factorization despite the fact that they represent usual candidates for positive invariance in relationship with linear time-invariant dynamics. The property of polyhedral factorization is recalled in the next proposition:

Proposition 1. There exists a regular ordered factorization for a polyhedral set:

$$
\Omega=\left\{x \in \mathbb{R}^{n}: F x \leq w\right\}
$$

described by its minimal half space representation, if there exists a block diagonalization of the matrix $F$ via a column permutation.

The relationship between $\mathscr{D}$-invariance and invariance in the extended state-space is formally stated in the next theorem without proof for brevity.

Theorem 1. The system (1) admits a regular convex $\mathscr{D}$-invariant set if and only if there exists an invariant set for the system:

$$
z(k)=\left[\begin{array}{cccc}
A_{1} & \ldots & A_{d-1} & A_{d} \\
I & \ldots & 0 & 0 \\
\vdots & \ddots & \vdots & \vdots \\
0 & \ldots & I & 0
\end{array}\right] z(k-1),
$$

which admits a regular ordered factorization.

Proposition 2. Let

$$
\Omega=\left\{x \in \mathbb{R}^{n d} \mid F x \leq w\right\}
$$

be an extended invariant set with respect to the system (2). A regular ordered factorization with dimension-n factors exists if there exists a transformation matrix $T \in \mathbb{R}^{(n d) \times(n d)}$ such that: 


$$
F T^{-1}=\left[\begin{array}{cccc}
F_{1} & 0 & \ldots & 0 \\
0 & F_{2} & \ddots & 0 \\
\vdots & \ddots & \ddots & \vdots \\
0 & \ldots & 0 & F_{d}
\end{array}\right]
$$

Corollary 1. Let a delay-difference equation be described by (1). There exists a $\mathscr{D}$ invariant set for this dynamical system in $\mathbb{R}^{n}$ if the following conditions are fulfiled:

- There exists a similarity transformation matrix $T$ such that

$$
\left[\begin{array}{cccc}
B_{1} & \ldots & B_{d-1} & B_{d} \\
I & \ldots & 0 & 0 \\
\vdots & \ddots & \vdots & \vdots \\
0 & \ldots & I & 0
\end{array}\right]=T\left[\begin{array}{cccc}
A_{1} & \ldots & A_{d-1} & A_{d} \\
I & \ldots & 0 & 0 \\
\vdots & \ddots & \vdots & \vdots \\
0 & \ldots & I & 0
\end{array}\right] T^{-1}
$$

- There exists an invariant set with respect to the system

$$
\tilde{z}\left(k=\left[\begin{array}{cccc}
B_{1} & \ldots & B_{d-1} & B_{d} \\
I & \ldots & 0 & 0 \\
\vdots & \ddots & \vdots & \vdots \\
0 & \ldots & I & 0
\end{array}\right] \tilde{z}(k-1)\right.
$$

which admits a regular ordered factorization.

\section{Existence and uniqueness of similarity transformations in the construction of $\mathscr{D}$-invariant sets}

We introduce in this section similarity transformations in the construction of $\mathscr{D}$ invariant sets all by preserving the dynamical model in the form of a delay difference equation in $\mathbb{R}^{n}$.

Sylvester equations play a central role in many areas of applied mathematics and in particular in systems and control theory. Before introducing formally these equations, we need to introduce first Schur's lemma.

Lemma 1. If $A$ is a square $n \times n$ matrix, then $A$ can be expressed as $A=Q U Q^{*}$. Where $Q^{*}$ is the trans-conjugate of the unitary matrix $Q\left(Q^{-1}=Q^{*}\right)$; $U$ is an upper triangular matrix (Schur form), containing the eigenvalues of A on its diagonal.

Let us consider the equation $A X+X B=C$ where $A \in M_{n}, B \in M_{m}$ and $C \in M_{n \times m}$, where $M_{n}$ denotes the set of square $n \times n$ matrices, and $M_{n \times m}$ denotes the set of $n \times m$ matrices. The Sylvester equation can be written in the form $\left(I_{m} \otimes A+B^{T} \otimes\right.$ $\left.I_{n}\right) * \operatorname{Vect}(X)=\operatorname{Vect}(C)$, where $\operatorname{Vect}(X)$ is the vertical concatenation of the columns of the matrix $X, I_{(.)}$is the identity matrix. $\otimes$ denotes the Kronecker product of two 
matrices. The spectrum of a matrix $A \in M_{n}$ is the set of the eigenvalues of $A$, denoted by $\lambda(A)$, while the spectral radius is defined as $\rho(A):=\max _{\xi \in \lambda(A)}(|\xi|)$.

$$
\begin{array}{r}
I_{m} \otimes A \in M_{m n} \\
B^{T} \otimes I_{n} \in M_{m n} .
\end{array}
$$

Theorem 2. If $\eta \in \lambda(A)$ and $v \in \mathbb{C}^{n}$ is the corresponding eigenvector of $A$, and if $\mu \in \lambda\left(B^{T}\right)$ and $w \in \mathbb{C}^{m}$ is the corresponding eigenvector of $B^{T}$, then $\eta+\mu$ is an eigenvalue of $I_{m} \otimes A+B^{T} \otimes I_{n}$ with $w \otimes v$ its corresponding eigenvector.

If $\lambda(A)=\left\{\eta_{1}, \eta_{2}, \ldots, \eta_{n}\right\}$ and $\lambda\left(B^{T}\right)=\left\{\mu_{1}, \mu_{2}, \ldots, \mu_{m}\right\}$, then:

$$
\lambda\left(I_{m} \otimes A+B^{T} \otimes I_{n}\right)=\left\{\eta_{i}+\mu_{j} ; i \in\{1, \cdots, n\}, j \in\{1, \cdots, m\}\right\} .
$$

Proof. The Schur decomposition of $A$ and $B^{T}$ is :

$A=Q_{A} U_{A} Q_{A}^{*}$ and $B^{T}=Q_{B^{T}} U_{B^{T}} Q_{B^{T}}^{*}$.

$Q_{A}^{*} Q_{A}=I_{n}, Q_{B^{T}}^{*} Q_{B^{T}}=I_{m}$.

$$
\left\{\begin{array}{r}
U_{A}=Q_{A}^{*} A Q_{A} \\
U_{B^{T}}=Q_{B^{T}}^{*} B^{T} Q_{B^{T}}
\end{array}\right.
$$

$U_{A}$ and $U_{B^{T}}$ in equation (16) are two upper triangular matrices. Let us now assume that $W$ is the Kronecker product $W=Q_{B^{T}} \otimes Q_{A} \in M_{m n}$, then:

$$
\begin{array}{r}
W^{*} W=\left(Q_{B^{T}} \otimes Q_{A}\right)^{*}\left(Q_{B^{T}} \otimes Q_{A}\right)=\left(Q_{B^{T}}^{*} \otimes Q_{A}^{*}\right)\left(Q_{B^{T}} \otimes Q_{A}\right) \\
=\left(Q_{B^{T}}^{*} Q_{B^{T}}\right) \otimes\left(Q_{A}^{*} Q_{A}\right)=I_{m n} .
\end{array}
$$

It holds also:

- $W^{*}\left(I_{m} \otimes A\right) W=\left(Q_{B^{T}}^{*} \otimes Q_{A}^{*}\right)\left(I_{m} \otimes Q_{A} U_{A} Q_{A}^{*}\right)\left(Q_{B^{T}} \otimes Q_{A}\right)=\left(Q_{B^{T}}^{*} \otimes U_{A} Q_{A}^{*}\right)\left(Q_{B^{T}} \otimes\right.$ $\left.Q_{A}\right)=\left(I_{m} \otimes U_{A}\right)$.

- $W^{*}\left(B^{T} \otimes I_{n}\right) W=\left(Q_{B^{T}}^{*} \otimes Q_{A}^{*}\right)\left(Q_{B^{T}} U_{B^{T}} Q_{B^{T}}^{*} \otimes I_{n}\right)\left(Q_{B^{T}} \otimes Q_{A}\right)=\left(U_{B^{T}} Q_{B^{T}}^{*} \otimes Q_{A}^{*}\right)\left(Q_{B^{T}} \otimes\right.$ $\left.Q_{A}\right)=\left(U_{B^{T}} \otimes I_{n}\right)$.

From the elements provided above, it becomes clear that:

$$
\begin{gathered}
W^{*}\left(I_{m} \otimes A+B^{T} \otimes I_{n}\right) W=I_{m} \otimes U_{A}+U_{B^{T}} \otimes I_{n} \\
=\left(\begin{array}{cccc}
U_{A} & 0 & 0 & 0 \\
0 & U_{A} & 0 & 0 \\
0 & 0 & \ddots & 0 \\
0 & 0 & 0 & U_{A}
\end{array}\right)+\left(\begin{array}{cccc}
\mu_{1} I_{n} & * & * & * \\
0 & \mu_{2} I_{n} & * & * \\
0 & 0 & \ddots & * \\
0 & 0 & 0 & \mu_{m} I_{n}
\end{array}\right) \\
=\left(\begin{array}{cccc}
\mu_{1} I_{n}+U_{A} & * & * & * \\
0 & \mu_{2} I_{n}+U_{A} & * & * \\
0 & 0 & \ddots & * \\
0 & 0 & 0 & \mu_{m} I_{n}+U_{A}
\end{array}\right)
\end{gathered}
$$


One can see that the diagonal elements of the resultant upper triangular matrix contain all sum pairs of eigenvalues of $U_{A}, U_{B^{T}}$

Remark 1. If for some $i$ and $j, \eta_{i}+\mu_{j}=0$, then rank of $\left(I_{m} \otimes A+B^{T} \otimes I_{n}\right)$ is strictly less than $n \times m$, then the solution for the system $\left(I_{m} \otimes A+B^{T} \otimes I_{n}\right) \operatorname{Vect}(X)=C$ is not unique.

Theorem 3. The Equation $A X+X B=C$ has a unique solution $X \in M_{n \times m}$ if and only if $\lambda(A) \cap \lambda(-B)=\emptyset$

Proof. Follows directly from Theorem 2.

In this paper, we are interested in the similarity transformation as an auxiliary tool for the construction of $\mathscr{D}$-invariant sets while preserving the dynamical model in the form of a delay difference equation in $\mathbb{R}^{n}$, i.e starting from an extended state space model of a given dynamical system, we obtain another extended state space model, which has the same dimension as the first one, via a simple change of coordinates. Such a similarity transformation represents a parametrization of the conditions for the existence of a regular ordered factorization.

In addition, one can see that solving the problem of determination of a matrix $\mathrm{T}$ in equation (13) is equivalent to the existence of an invertible matrix $\mathrm{T}$ which verifies a particular (homogeneous) Sylvester equation. In Theorem 2 it is shown that the equation (13) can be rewritten as a linear (in our case homogeneous) equation of size $(n d)^{2} \times(n d)^{2}$. This equation has non-trivial solutions if it is singular which is equivalent to matrices $\mathrm{A}$ and $\mathrm{B}$ having at least one common eigenvalue. It is clear that equation (13) represents a similarity transformation and that matrices $\mathrm{A}$ and $\mathrm{B}$ share the same set of eigenvalues. Subsequently, applying Theorem 3 guarantees that the transformation exists and more than that, it is not unique. In fact, the solution, in vectorial form, is the full null space of the matrix $\left(I_{n d} \otimes B-A^{T} \otimes I_{n d}\right)$.

The similarity transformation corresponds to a transformation of the state $\tilde{z}=T z$, where $\tilde{z}$ and $z$ are the state vectors of the extended state space realization. This results in several possible canonical forms. Different properties stand out more clearly in different realizations, and some forms may have advantages in some applications (recall for example the controllable and observable canonical forms in classical control theory). It is worth mentioning that most of dynamical properties of an LTI system, such as input-output properties and the impulse response and so on, are not changed by similarity transformations.

Remark 2. In general, algebraic equivalence ${ }^{1}$ does not preserve stability properties of a dynamical system [19,20], and for this a necessary and sufficient condition will be the topological equivalence, which is the algebraic condition plus the condition on the Euclidean norm of the matrix $T$ [18].

In our case and since we are working in a time-invariant setting, it follows from [18] that two LTI systems are strictly equivalent whenever their phase vectors are related

\footnotetext{
${ }^{1}$ See [18] for a formal definition of algebraic equivalence.
} 
for all time $t$ as $(t, \tilde{z})=(t, T z)$, where $T$ is a nonsingular constant matrix, and obviously, strict equivalence implies topological equivalence.

When dealing with scalar systems (one state only), with simple linear algebra manipulations, it can be shown that the constraint imposed on the similarity transformation is very restrictive and allow only scaling type of change of coordinates on the original delay difference equation, without an impact on the regular ordered factorization.

Example 1. For illustration, let us consider dynamical matrix

$$
A=\left[\begin{array}{cccc}
a_{1} & \ldots & a_{d-1} & a_{d} \\
1 & \ldots & 0 & 0 \\
\vdots & \ddots & \vdots & \vdots \\
0 & \ldots & 1 & 0
\end{array}\right]
$$

there exists an infinite number of combinations of similarity transformations $\mathrm{T}$, which satisfy equality (13). All matrices $\mathrm{T}$ are generated from the null space of the matrix $\left(I_{d} \otimes A-B^{T} \otimes I_{d}\right)$. This null space is the same as the one of $\left(I_{d} \otimes A-A^{T} \otimes I_{d}\right)$ since we consider here only scalar systems. For $d=2$, the null space is generated by the two matrices:

$$
\left\{\left[\begin{array}{ll}
1 & 0 \\
0 & 1
\end{array}\right],\left[\begin{array}{cc}
a_{0} / a_{1} & 1 \\
1 / a_{1} & 0
\end{array}\right]\right\}
$$

while for $d=3$, the null space is generated by the three matrices:

$$
\left\{\left[\begin{array}{lll}
1 & 0 & 0 \\
0 & 1 & 0 \\
0 & 0 & 1
\end{array}\right],\left[\begin{array}{ccc}
a_{0} / a_{2} & a_{1} / a_{2} & 1 \\
1 / a_{2} & 0 & 0 \\
0 & 1 / a_{2} & 0
\end{array}\right],\left[\begin{array}{ccc}
a_{1} / a_{2} & 1 & 0 \\
0 & a_{1} / a_{2} & 0 \\
1 / a_{2} & -a_{0} / a_{2} & 0
\end{array}\right]\right\} .
$$

Next we make a step forward towards the study of the structure of all matrices $T$ which allow transformations by maintaining the dynamical model in the class of a DDE in $\mathbb{R}^{n}$. Specifically, we will be interested in the present work in the case of systems with two states $n=2$ and a maximum delay $d=2$ in (1).

Let us consider the extended dynamical system (2) in this case:

$$
z(k)=\left[\begin{array}{cc}
A_{1} & A_{2} \\
I & 0
\end{array}\right] z(k-1) \Leftrightarrow x(k)=A_{1} x(k-1)+A_{2} x(k-2)
$$

After the change of coordinates, in the novel basis:

$$
\tilde{z}(k)=\left[\begin{array}{cc}
B_{1} & B_{2} \\
I & 0
\end{array}\right] \tilde{z}_{k}=T\left[\begin{array}{cc}
A_{1} & A_{2} \\
I & 0
\end{array}\right] T^{-1} \tilde{z}(k-1)
$$

The relationship between the augmented state in the two basis of coordinates is:

$$
\tilde{z}(k)=T z(k)
$$




$$
\begin{gathered}
{\left[\begin{array}{c}
\tilde{x}(k) \\
\tilde{x}(k-1)
\end{array}\right]=\left[\begin{array}{ll}
T_{11} & T_{12} \\
T_{21} & T_{22}
\end{array}\right]\left[\begin{array}{c}
x(k) \\
x(k-1)
\end{array}\right]} \\
\tilde{z}(k)=\left[\begin{array}{cc}
B_{1} & B_{2} \\
I & 0
\end{array}\right] \tilde{z}(k-1) \Leftrightarrow \tilde{x}(k)=B_{1} \tilde{x}(k-1)+B_{2} \tilde{x}(k-2) \\
T_{21} x(k)+T_{22} x(k-1)=T_{11} x(k-1)+T_{12} x(k-2) \\
T_{21} x(k)=\left(T_{11}-T_{22}\right) x(k-1)+T_{12} x(k-2) \\
x(k)=A_{1} x(k-1)+A_{2} x(k-2)
\end{gathered}
$$

From equation (25), we can easily derive the matrix $T$ :

- If $T_{21}=\mathbf{O} \rightarrow T_{11}=T_{22}=T_{*}$ and $T_{12}=\mathbf{O}$

$$
T=\left[\begin{array}{cc}
T_{*} & \mathbf{O} \\
\mathbf{O} & T_{*}
\end{array}\right]
$$

- If $T_{21}=I_{2 \times 2} \rightarrow T_{12}=A_{2}$ and $T_{11}=A_{1}+T_{22}$

$$
T=\left[\begin{array}{cc}
A_{1}+T_{22} & A_{2} \\
I_{2 \times 2} & T_{22}
\end{array}\right]
$$

- If $T_{21} \neq I_{2 \times 2}$ and is invertible

$$
\begin{gathered}
\left\{\begin{array} { c } 
{ A _ { 1 } = T _ { 2 1 } ^ { - 1 } ( T _ { 1 1 } - T _ { 2 2 } ) } \\
{ A _ { 2 } = T _ { 2 1 } ^ { - 1 } T _ { 1 2 } }
\end{array} \Leftrightarrow \left\{\begin{array}{r}
T_{12}=T_{21} A_{2} \\
T_{11}=T_{22}+T_{21} A_{1}
\end{array}\right.\right. \\
T=\left[\begin{array}{cc}
T_{22}+T_{21} A_{1} & T_{21} A_{2} \\
T_{21} & T_{22}
\end{array}\right]
\end{gathered}
$$

Remark 3. Note that the first case when $T_{21}=\mathbf{O}$, and the second one $T_{21}=I_{2 \times 2}$ represent particular structures of a broader family of matrices $T$ presented in the third case by relaxing the invertibility assumption for $T_{21}$. Just by setting $T_{21}=\mathbf{O}$ then $T_{21}=I_{2 \times 2}$, equation (3) take the form of equations (26) then (27) respectively. All transition matrices for (25) are thus generated by the two matrices:

$$
\left\{\left[\begin{array}{ll}
I & 0 \\
0 & I
\end{array}\right],\left[\begin{array}{cc}
A_{1} & A_{2} \\
I & 0
\end{array}\right]\right\}
$$

Based on the above particular forms, matrix $T$ can be written, using the Kronecker product, in a compact form.

$$
T=\left(I_{2 \times 2} \otimes T_{22}\right)+\left(I_{2 \times 2} \otimes T_{21}\right) A
$$

It is worth noting that square matrices $T_{21}$ and $T_{22}$ can be chosen arbitrarily as long as they lead to an invertible matrix $T$. 


\section{Transformation allowing regular ordered factorization}

Let us consider the change of basis $\tilde{z}=T z$ applied to the same dynamical system (20). For sake of simplicity, we examine in this section transformation of the form: $z=T^{-1} \tilde{z}=\Gamma \tilde{z}$, then $\Gamma$ can be written as:

$$
\Gamma=\left[\begin{array}{cc}
\gamma_{22}+\gamma_{21} B_{1} & \gamma_{21} B_{2} \\
\gamma_{21} & \gamma_{22}
\end{array}\right]
$$

which is equivalent to :

$$
\Gamma=\left[\left(I_{2 \times 2} \otimes \gamma_{22}\right)+\left(I_{2 \times 2} \otimes \gamma_{21}\right) B\right]
$$

Let $\Omega=\left\{x \in \mathbb{R}^{4} \mid F x \leq w\right\}$ be an invariant set in the extended state space with respect to the dynamical system (20), then $\tilde{\Omega}=\left\{\tilde{x} \in \mathbb{R}^{4} \mid F \Gamma \tilde{x} \leq w\right\}$ will be the corresponding invariant set for the extended state realization (24).

$$
\begin{gathered}
F \Gamma=F\left[\left(I_{2 \times 2} \otimes \gamma_{22}\right)+\left(I_{2 \times 2} \otimes \gamma_{21}\right) B\right] \\
=F\left(I_{2 \times 2} \otimes \gamma_{22}\right)+F\left(I_{2 \times 2} \otimes \gamma_{21}\right) B \\
\tilde{\Omega}=\left\{\tilde{x} \in \mathbb{R}^{4} \mid\left[F\left(I_{2 \times 2} \otimes \gamma_{22}\right)+F\left(I_{2 \times 2} \otimes \gamma_{21}\right) B\right] \tilde{x} \leq w\right\} \\
F \Gamma=\left[F\left[\begin{array}{cc}
\gamma_{22} & 0_{2 \times 2} \\
0_{2 \times 2} & \gamma_{22}
\end{array}\right]+F\left[\begin{array}{cc}
\gamma_{21} & 0_{2 \times 2} \\
0_{2 \times 2} & \gamma_{21}
\end{array}\right] B\right]
\end{gathered}
$$

Let $\mathrm{F}$ be:

$$
F=\left[\begin{array}{ll}
F_{1} & F_{2} \\
F_{3} & F_{4}
\end{array}\right] \text { then } F \Gamma=\left[\begin{array}{ll}
F_{1}\left(\gamma_{22}+\gamma_{21} B_{1}\right)+F_{2} \gamma_{21} & F_{2} \gamma_{22}+F_{1} \gamma_{21} B_{2} \\
F_{3}\left(\gamma_{22}+\gamma_{21} B_{1}\right)+F_{4} \gamma_{21} & F_{4} \gamma_{22}+F_{3} \gamma_{21} B_{2}
\end{array}\right]
$$

The necessary and sufficient condition for the existence of a factorization for the invariant set is that the matrix $F \Gamma$ has a lower triangular structure:

$$
F \Gamma=\left(\begin{array}{cc}
* & 0 \\
* & *
\end{array}\right)
$$

which means that there exists $\gamma_{22}$ and $\gamma_{21}$ verifying the equality:

$$
F_{2} \gamma_{22}+F_{1} \gamma_{21} B_{2}=0
$$

\section{Illustrative Example}

Let us consider the following dynamical system:

$$
x(k)=A_{1} x(k-1)+A_{2} x(k-2),
$$




$$
\begin{gathered}
A_{1}=\left[\begin{array}{cc}
-0.5026 & 1.3088 \\
0.5201 & 0.9026
\end{array}\right] \\
A_{2}=\left[\begin{array}{cc}
-0.059 & 0.4517 \\
-0.0935 & -0.7510
\end{array}\right]
\end{gathered}
$$

The necessary condition for the existence of a $\mathscr{D}$-contractive set proposed in [13] is not fulfilled. One can verify that the spectral radius of $A_{1}$ is not subunitary, $\rho\left(A_{1}\right)=$ $1.2837>1$. More than that, the necessary condition proposed in [21] is not verified. We can easily verify that the spectral radius of the sum $\rho\left(A_{1}+A_{2}\right)=1.1422>1$, and the set of generalized eigenvalues possesses four elements on the unit circle:

$$
\begin{array}{r}
\gamma(U, V)=0.4611 \pm 0.8873 \mathrm{i}, 0.6392 \pm 0.7690 \mathrm{i}, \\
0.144,-0.096,-10.410,6.943 .
\end{array}
$$

The delay difference equation (38) does not admit a $\mathscr{D}$-invariant set. Note that the extended state space representation has a strictly stable transition matrix, which allows the construction of invariant set $\Omega_{A_{e}} \subset \mathbb{R}^{4}$.

$$
z(k)=\left[\begin{array}{cccc}
-0.5026 & 1.3088 & -0.059 & 0.4517 \\
0.5201 & 0.9026 & -0.0935 & -0.7510 \\
1 & 0 & 0 & 0 \\
0 & 1 & 0 & 0
\end{array}\right] z(k-1) .
$$

Let $\Omega_{A_{e}}=\left\{x \in \mathbb{R}^{4} \mid F x \leq w\right\}$ be the extended invariant set with respect to (41). With linear algebra manipulations, we can find a similarity transformation $\mathrm{T}$ such that $(\tilde{z}=T z)$, which allows formulation the system $z(k)=A z(k-1)$ in the equivalent form $\tilde{z}(k)=B \tilde{z}(k-1)$, and there exists an invariant set with respect to this last dynamical system which admits a regular ordered factorization in $\mathbb{R}^{2}$. Let us take the transition matrix $T=\left(I_{2 \times 2} \otimes T_{22}\right)+\left(I_{2 \times 2} \otimes T_{21}\right) A$,

$$
\begin{aligned}
& T_{21}=\left[\begin{array}{cc}
-28.729 & 3.932 \\
30.667 & -3.549
\end{array}\right] \\
& T_{22}=\left[\begin{array}{cc}
-3.161 & 34.342 \\
4.831 & -37.413
\end{array}\right] .
\end{aligned}
$$

and

$$
T=\left[\begin{array}{cccc}
13.322 & 0.289 & 1.326 & -15.930 \\
-12.427 & -0.479 & -1.476 & 16.517 \\
-28.729 & 3.932 & -3.161 & 34.342 \\
30.667 & -3.549 & 4.831 & -37.413
\end{array}\right]
$$

The dynamical system in the new basis:

$$
\tilde{z}(k)=B \tilde{z}(k-1)=T A T^{-1} \tilde{z}(k-1),
$$

with 


$$
B=\left[\begin{array}{cccc}
0.20 & -0.34 & 0.24 & -0.17 \\
0.34 & 0.20 & 0.17 & 0.24 \\
1 & 0 & 0 & 0 \\
0 & 1 & 0 & 0
\end{array}\right]
$$

has a strictly stable transition matrix, which has the same set of eigenvalues as the system (41).

$$
\lambda(A)=\lambda(B)=0.6108 \pm 0.3697 \mathrm{i},-0.4108 \pm 0.0297 \mathrm{i} .
$$

This allows the construction of an invariant set $\Omega_{B_{e}} \subset \mathbb{R}^{4}$ which is factorizable, then the delay difference equation $\tilde{x}(k)=B_{1} \tilde{x}(k-1)+B_{2} \tilde{x}(k-2)$ admits a $\mathscr{D}$-invariant set $\Omega \subset \mathbb{R}^{2}$ in this novel basis. It can be shown that this particular choice of $T_{21}$ and $T_{22}$ verifies (37).

$$
\begin{gathered}
\Omega=\left\{\tilde{x} \in \mathbb{R}^{2} \mid\left[\begin{array}{cc}
\sqrt{2} & -\sqrt{2} \\
-\sqrt{2} & \sqrt{2} \\
\sqrt{2} & \sqrt{2} \\
-\sqrt{2} & -\sqrt{2}
\end{array}\right] \tilde{x} \leq\left[\begin{array}{l}
2 \\
2 \\
2 \\
2
\end{array}\right]\right\} \\
\Omega_{B_{e}}=\left\{\tilde{z} \in \mathbb{R}^{4} \mid\left[\begin{array}{cccc}
\sqrt{2} & -\sqrt{2} & 0 & 0 \\
-\sqrt{2} & \sqrt{2} & 0 & 0 \\
\sqrt{2} & \sqrt{2} & 0 & 0 \\
-\sqrt{2} & -\sqrt{2} & 0 & 0 \\
0 & 0 & \sqrt{2} & -\sqrt{2} \\
0 & 0 & -\sqrt{2} & \sqrt{2} \\
0 & 0 & \sqrt{2} & \sqrt{2} \\
0 & 0 & -\sqrt{2} & -\sqrt{2}
\end{array}\right] \tilde{z} \leq\left[\begin{array}{l}
2 \\
2 \\
2 \\
2 \\
2 \\
2 \\
2 \\
2
\end{array}\right]\right\}
\end{gathered}
$$

Figure 1 presents the $\mathscr{D}$-invariant set obtained. Dashed black line represents the state trajectory starting from the initial states $x(-1)=[0,-\sqrt{2}]^{\prime}$ and $x(-2)=[0,0]^{\prime}$ with respect to the DDE $\tilde{x}(k)=B_{1} \tilde{x}(k-1)+B_{2} \tilde{x}(k-2)$. However, dashed red line represents the state trajectory starting from the same initial states with respect to the DDE (38).

Dashed lines in Figure 2 represent the state trajectories starting from the same initial state in different basis. We can see that the state trajectory in the original basis is not monotone. Thus, the yellow set is not $\mathscr{D}$-invariant with respect to the dynamics (38). However, in the new basis, the trajectory is converging monotonically inside the blue set which is $\mathscr{D}$-invariant with respect to the dynamic $\tilde{x}(k)=B_{1} \tilde{x}(k-1)+$ $B_{2} \tilde{x}(k-2)$. 


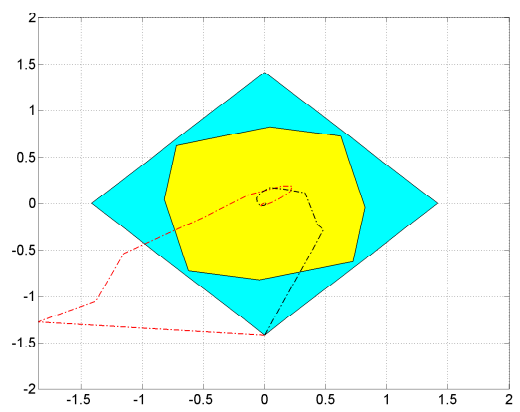

Fig. 1 Set $\Omega$ in cyan and the set iterate $B_{1} \Omega \oplus B_{2} \Omega$ in yellow
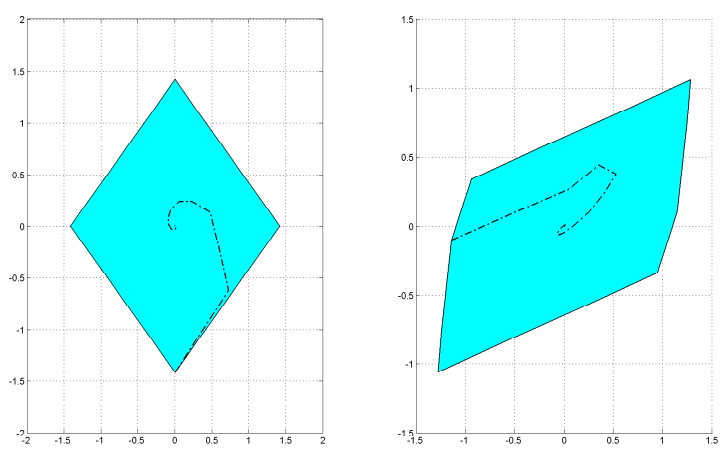

Fig. 2 State trajectories starting from the same initial state and the corresponding $\mathscr{D}$-invariant set (left) and the projection of $\Omega_{A_{e}}$

\section{Conclusion}

A unifying characterization of the link between invariance in the extended state space and $\mathscr{D}$-invariance, via set factorization, was studied for discrete-time DDEs. Low complexity invariant sets were recalled and it was shown that set factorization combined with similarity transformations allow a flexible description of invariant sets in state spaces of same dimension. Thus, a relaxation of the conservativeness of the existing $\mathscr{D}$-invariance constructions was delivered for a more flexible $\mathscr{D}$ invariance characterization. 
Title Suppressed Due to Excessive Length

\section{References}

1. D. Mayne, J. Rawlings, C. Rao, and P. Scokaert, "Constrained model predictive control: Stability and optimality," Automatica, vol. 36, pp. 789-814, 2000.

2. S. Olaru, J. A. De Dona, M. M. Seron, and F. Stoican, "Positive invariant sets for fault tolerant multisensor control schemes," International Journal of Control, vol. 83, no. 12, pp. 26222640, 2010.

3. F. Stoican and S. Olaru, Set-theoretic Fault-tolerant Control in Multisensor Systems. John Wiley \& Sons, 2013.

4. G. Bitsoris, "Positively invariant polyhedral sets of discrete-time linear systems," International Journal of Control, vol. 47, no. 6, pp. 1713-1726, 1988.

5. E. Gilbert and K. Tan, "Linear systems with state and control constraints, the theory and application of maximal output admissible sets," IEEE Transaction on Automatic Control, vol. 36, pp. 1008-1020, 1991.

6. F. Blanchini and S. Miani, Set-theoretic methods in control. Boston: Birkhuser, 2008.

7. R. H. Gielen, M. Lazar, and I. V. Kolmanovsky, "Lyapunov methods for time-invariant delay difference inclusions," SIAM Journal on Control and Optimization, vol. 50, no. 1, pp. 110132, 2012.

8. M. T. Laraba, S. Olaru, S.-I. Niculescu, F. Blanchini, S. Miani, D. Casagrande, and G. Giordano, "Guide on set invariance for delay difference equations," Annual Reviews in Control, vol. 41.

9. W. Lombardi, S. Olaru, M. Lazar, and S.-I. Niculescu, "On positive invariance for delay difference equations," in Proceedings of the IEEE American Control Conference, 2011, pp. 36743679 .

10. W. Lombardi, A. Luca, S. Olaru, and S.-I. Niculescu, "On the polyhedral set invariance conditions for time-delay systems," in Proceedings of the 18th IFAC World Congress, Milano, Italy, 2011, pp. 308-313.

11. M. T. Laraba, S. Olaru, S.-I. Niculescu, F. Blanchini, S. Miani, D. Casagrande, and G. Giordano, "Set invariance for delay difference equations," in 12th IFAC Workshop on Time Delay Systems, Ann Arbor (MI), USA, 2015.

12. S. Olaru, N. Stanković, G. Bitsoris, and S.-I. Niculescu, "Low complexity invariant sets for time-delay systems: A set factorization approach," in Low-Complexity Controllers for TimeDelay Systems. Springer, 2014, pp. 127-139.

13. W. Lombardi, "Constrained control for time-delay systems." Ph.D. dissertation, Supélec, 2011.

14. W. Lombardi, S. Olaru, G. Bitsoris, and S.-I. Niculescu, "Cyclic invariance for discrete timedelay systems," Automatica, vol. 48, no. 10, pp. 2730-2733, 2012.

15. S. V. Raković, R. H. Gielen, and M. Lazar, "Construction of invariant families of sets for linear systems with delay," in Proceedings of the IEEE American Control Conference, 2012, pp. 6246-6251.

16. N. Halbwachs, D. Merchat, and C. Parent-Vigouroux, "Cartesian factoring of polyhedra in linear relation analysis," in Cousot R.(ed.) Static Analysis Springer Series Lecture Notes in Computer Science, no. 2694, pp. 355-365, 2003.

17. N. Halbwachs, D. Merchat, and L. Gonnord, "Some ways to reduce the space dimension in polyhedra computations," Formal Methods Syst. Des, vol. 29, no. 1, pp. 79-95, 2006.

18. R. E. Kalman, "Mathematical description of linear dynamical systems," J.S.I.A.M. Control, vol. 1, no. 2, pp. 152-192, 1963.

19. R. E. Kalman and J. E. Bertram, "Control system analysis and design via the second method of Lyapunov," J. Basic Engr. (Trans. A.S.M.E., no. 82, pp. 371-393, 1960.

20. W. Hahn, "Theorie und anwendung der direkten methode yon Ljapunov." Springer, 1959.

21. N. Stankovic, S. Olaru, and S. I. Niculescu, "Further remarks on asymptotic stability and set invariance for linear delay difference equations," Automatica, vol. 50, no. 8, pp. 2191-2195, 2014. 